\title{
On Coexistence with COVID-19: Estimations and Perspectives
}

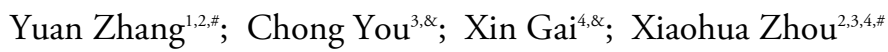

Given the harsh reality faced by the global effort to contain coronavirus disease 2019 (COVID-19) and the virtual impossibility of its worldwide eradication in the foreseeable future, global human coexistence with fast mutating severe acute respiratory syndrome coronavirus 2 (SARS-CoV-2) may have to occur, for the time being, irrespective of the wishes and aspirations of our people. Therefore, for China, the risk associated with small to medium scale outbreaks induced by imported cases and the corresponding need to continuously and promptly suppress domestic infections would persist for a certain period of time. However, such reality would not imply that we can deviate from our existing effective COVID-Zero strategy on entry-exit quarantine measures and community non-pharmaceutical interventions (NPIs) containment measures and embrace certain "open-up" strategies without reservation, resting solely on the hypothesis of the success of herd immunity induced by vaccination advocated by certain western countries. In this article, we found that even in a highly underestimated outbreak scenario under the most optimistic assumptions, once China adopts the control and prevention strategies of some typical western countries, the number of the daily new confirmed cases in China would likely rise up to hundreds of thousands of cases, and among which $>10,000$ cases would present with severe symptoms. Particularly, the number of standing severe cases would exceed the peak number nationwide in early 2020 within 1-2 days, which would have a devastating impact on the medical system of China and cause a great disaster within the nation.

\section{Estimation on A Lower Bound of The Number of Newly Reported Cases}

In this article, we used the real-world pandemic scenario in the following countries: the United States, the United Kingdom, Israel, Spain, and France, as a reference group to evaluate the corresponding potential consequences if pandemic response strategies in the aforementioned countries were to be adopted in China.
In order to obtain a conservative estimation/lower bound, we hereby recalled a series of census data and key epidemiology characteristics of each of these countries below.

The population of the mainland of China is $1,411,778,724$ based on the 7 th National Census (1), which yields a countrywide population density of 147 people/ $\mathrm{km}^{2}(1-2)$. In particular, the population of the eastern region, which includes the provincial-level administrative divisions (PLADs) of Hebei, Shandong, Jiangsu, Zhejiang, Fujian, Guangdong, Hainan, Beijing, Tianjin, and Shanghai accounts for $39.9 \%$ of the total population, which is $563,300,220$ (1), yielding a much higher population density of 661 people $/ \mathrm{km}^{2}$ (1,3-4). According to the latest report (5), a population of $777,046,000$ has been fully vaccinated in the mainland of China, which represents a full vaccination ratio of roughly $55.04 \%$. Natural immunity ratio is negligible in China considering the small number of cases with respect to its huge population size (6). The epidemiological information of the countries in our reference group has been collected and summarized in Table 1 .

Note that the cumulative number of confirmed cases can serve as a very conservative estimate on the population with natural immunity. Thus, we can see that for each country in the reference group, their vaccination coverage together with natural immunity ratio are higher than in China. As for the population density, the United States, Spain, and France all have population densities lower than China. At the same time, none of these reference countries have their population densities as high as that of the eastern region in China.

For now, we assumed that the immunity induced by inactivated vaccines used in China is as strong as the one induced by vaccines used in the reference countries and also as strong as natural immunity in terms of providing protection against infection/symptoms. The following over-optimistic assumptions were used: 1) the vaccination rate in China is as high as the reference countries; and 2) the population density of China or China's eastern region is as low as the reference 
TABLE 1. Epidemiology profiles of the reference countries.

\begin{tabular}{ccccrr}
\hline $\begin{array}{c}\text { Reference } \\
\text { country }\end{array}$ & Population & Population density & Vaccination ratio-full & $\begin{array}{c}\text { Confirmed cases- } \\
\text { cumulative }\end{array}$ & $\begin{array}{c}\text { Confirmed cases- } \\
\text { averaged in 7 days }\end{array}$ \\
\hline US & $332,580,000(7)$ & $36.2(8)$ & $51.16 \%(08 / 23)(9)$ & $37,939,641(10)$ & $150,098(10)$ \\
UK & $67,081,000(11)$ & $280.6(8)$ & $61.59 \%(08 / 22)(9)$ & $6,555,419(10)$ & $32,843(10)$ \\
Israel & $9,289,800(12)$ & $400.0(8)$ & $62.94 \%(08 / 23)(9)$ & $999,110(10)$ & $7,293(10)$ \\
Spain & $47,330,000(13)$ & $93.7(8)$ & $67.26 \%(08 / 22)(9)$ & $4,794,352(10)$ & $10,727(10)$ \\
France & $67,347,241(14)$ & $119.2(8)$ & $55.57 \%(08 / 22)(9)$ & $6,708,163(10)$ & $21,667(10)$ \\
\hline
\end{tabular}

countries (see detailed explanation below) if pandemic response strategies and community activity patterns in the reference countries were adopted in China, the ratio of infection would be similar to these countries. While in reality, such a rate should be much higher.

According to the dynamic transmission models in epidemiology, for example the well-known susceptibleinfected-removed (SIR) model and its variants, the daily increment in pandemic size can be estimated by $\beta S I / N$, where $\beta$ is the transmission rate, $S$ is the susceptible population, $I$ is the infected population and $N$ is the total population. If $\beta$ and the ratio of infection $I / N$ are fixed, the number of new infections is proportional to $S$, which is in turn approximately proportional to the population size $N$.

We could hence scale the size of $S$ to estimate the daily confirmed cases in China if the same strategies in the reference countries would have been adopted, under the aforementioned assumptions. Note that the estimates would be most likely over-optimistic due to the nature of assumptions (1-2), but still serve their purpose of obtaining a plausible lower bound of pandemic sizes.

To ensure that assumption (2) was always a conservative one and thus led to a universally overoptimistic estimate if China adopted response strategies of all the reference countries, we estimated various lower bounds of national-wide daily new infection sizes using the national population if the corresponding reference countries have a population density lower than China (i.e., the United States, Spain, and France). Otherwise, the lower bounds of daily new infection sizes in the eastern region were calculated using only the population within that part of China (i.e., Britain and Israel). Recall that the population in the eastern region of China is significantly higher than any of the reference countries, ensuring that assumption (2) remains conservative. Estimations of the lower bound in the two scenarios above are precisely calculated in the following Equations:

(i) Population density of reference country < Overall population density of China

$$
\begin{aligned}
& \text { lowerboundestimate }=\frac{\text { Population of China }}{\text { Population of Reference Country }} \\
& \times \bar{C} \text { (Reference Country) }
\end{aligned}
$$

(ii) Population density of reference country > Overall population density of China

$$
\begin{aligned}
& \text { lowerboundestimate }=\frac{\text { Population of Easternregion }}{\text { Population of Reference Country }} \times \\
& \bar{C}(\text { Reference Country })
\end{aligned}
$$

Remark 1: In Equation (1)-(2), $\bar{C}$ (Reference Country) stands for the average of confirmed cases reported in the reference country, August 17-August 23, 2021.

Based on Equation (1)-(2), the conservative estimates on hypothetic daily new infections in China can be obtained under strategies in the reference countries in Table 2. We can see that even with the aforementioned over-optimistic assumptions, the size of daily new infection in China would be very likely of hundreds of thousands if China adopted the strategies in these reference countries.

\section{Estimation on a Lower Bound of Daily New Severe Cases}

There have been recent arguments that a higher infection size is now more tolerable provided that severe cases of COVID-19 have been substantially reduced under the large-scale vaccination campaign, preventing the medical system from being overrun. While vaccination has been proved to significantly reduce the development of severe symptoms after infection from the original and mutated strain of the virus, the emergence of new mutated strains would potentially disrupt such protection. In order to depict the combined effect from vaccine coverage rate, vaccine protection efficiency, and the characteristics of the Delta strain on the current rate of severe cases, we used real-world data in the most recent outbreak in Yangzhou, China and modified it based on nationwide age structure.

By midnight of August 23, 2021, there have been a 
TABLE 2. Estimates on the lower bound of daily new infections in China under strategies in the reference countries.

\begin{tabular}{cccc}
\hline Reference country & $\begin{array}{c}\text { Population in the } \\
\text { reference country }\end{array}$ & $\begin{array}{c}\text { Confirmed cases-averaged in 7 days in } \\
\text { the reference country }\end{array}$ & $\begin{array}{c}\text { Estimated lower bound of daily new } \\
\text { cases in China }\end{array}$ \\
\hline US & $332,580,000$ & 150,098 & 637,155 \\
UK & $67,081,000$ & 32,843 & 275,793 \\
Israel & $9,289,800$ & 7,293 & 442,221 \\
Spain & $47,330,000$ & 10,727 & 319,969 \\
France & $67,347,241$ & 21,667 & 454,198 \\
\hline
\end{tabular}

total of 568 cases reported in Yangzhou (15). Moreover, as of August 16, the city had 27 standing severe cases and 17 critical cases (16) Thus, the cumulative number of cases who had ever developed severe symptoms is at least $44(27+17)$, which yields a ratio of $7.7 \%$. Note that the outbreak in Yangzhou was still in a moderate scale without medical overloading, the observed rate can be considered as an optimistic estimate on the rate of severe cases for larger scale outbreaks.

However, it is worth noting that the outbreak in Yangzhou was mainly induced by the spread of COVID-19 in Mahjong clubs and that the elderly accounted for $41.0 \%$ of the total reported infections. Noting that the elderly is known to have a higher risk of developing severe symptoms, we had to rescale the rate of severe cases by adjusting the age structure in Yangzhou to match that of the whole population of China as follows.

The rate of severe cases in Yangzhou can be expressed as

$$
\rho^{s}=\rho_{1} p_{1}^{s}+\rho_{2} p_{2}^{s}=7.7 \%,
$$

where $\rho^{s}$ is the rate of severe cases in Yangzhou, $\rho_{1}=1-\rho_{2}=41.0 \%$ is the proportion of elderly in total infection in Yangzhou while $p_{1}^{s}$ and $p_{2}^{s}$ are the unknown rates of severe cases for elderly and non-elderly. In a same way, the rate of severe cases in the whole population of China can be expressed as

$$
q^{s}=q_{1} p_{1}^{s}+q_{2} p_{2}^{s},
$$

where $q$ is the rate of severe cases over the whole Chinese population, $q_{1}=1-q_{2}$ is the proportion of elderly in total infection in China. Under the massive outbreak hypothesized in the previous section, we assumed that $q_{1}$ equals the proportion of the elderly in the population in China, that is $18.7 \%$ (17), we have

$$
q^{s}=q_{1} p_{1}^{s}+q_{2} p_{2}^{s} \geq \frac{18.7}{41}\left(\rho_{1} p_{1}^{s}+\rho_{2} p_{2}^{s}\right)=3.5 \% .
$$

Note that lower bound was calculated based on a reasonable assumption on $p_{1}^{s}>p_{2}^{s}$. Here, we took $3.5 \%$ to be a reasonable lower bound for the rate of severe cases under the combined effects of current population age structure, vaccination coverage and protection rate, medical resources, and the characteristics of the Delta strain.

Remark 2: Considering the possibly lower vaccination coverage, protection, and the immunity to COVID-19 in elderly group, as well as higher aging level in eastern region of China, the aforementioned lower bound is likely to be underestimated again.

Remark 3: As most of the reference countries do not conduct community-level screening and contact tracing to the same lengths and strength as in China, the ascertainment rate is probability lower than that in Yangzhou (many asymptomatic/mild cases detected in Yangzhou might not be ascertainable in the reference countries). This implies that the rate of severe cases would be higher when applying the criterion of confirmed cases found in the reference countries to the reported cases in Yangzhou, and hence the fraction of severe cases was likely to be underestimated.

Note that all the aforementioned estimates are uniformly on the conservative side. Combining these over-optimistic estimates, we found the number of daily new severe cases or sickbed demand was as follows in Table 3 if we adopt the "open-up" strategies of these reference countries. We can see if strategies in these reference countries were adopted, the number of severe cases might exceed the peak of standing severe cases nation-wide in early 2020 [11,977, on February 18,2020 (18)] within 1-2 days, and thus would pose an unaffordable burden to the medical system in this country.

\section{DISCUSSION}

How to safely open up is one of the most interesting issues at present, which has been discussed in many studies (19-20). Among which, Foo et al. proposed four insightful key tenets for the safe transition from elimination strategies to open-up strategies: 1) retain flexible NPIs based on the changing epidemiology and hospital capacities; 2) maximize vaccination coverage; 3) shield industries and vulnerable groups from the 
TABLE 3. Estimates on the lower bound of daily severe cases in China under strategies in the reference countries.

\begin{tabular}{cc}
\hline Reference country & $\begin{array}{c}\text { Estimated lower bound of daily severe } \\
\text { cases in China }\end{array}$ \\
\hline US & 22,364 \\
UK & 9,680 \\
Israel & 15,522 \\
Spain & 11,231 \\
France & 15,942 \\
\hline
\end{tabular}

unintended consequences resulting from NPIs; and 4) detect and isolate COVID-19 promptly using extensive surveillance and stronger community social responsibility. However, due to the large population and relatively scarce health resources per capita in China, it is difficult to fully achieve tenets 1,3 , and 4, especially during a large-scale outbreak; therefore, China needs to be cautious about the decision on the open-up. Nevertheless, our suggestions towards China might not necessarily be applicable to other countries.

To be noted again, our results were based on elementary arithmetic calculations to provide quick estimates on the lower bound of the daily new infections and severe cases should China adopt coexistent or open-up policies seen in the reference countries. As a result, there were some limitations in our study, for example: 1) the estimates here were only the conservative lower bounds of, and not the actual number of cases, which is not possible to predict, but the order of magnitude seems reasonable; and 2) the effects on different vaccination strategies were not able to be incorporated into the estimations. More sophisticated dynamic models are needed to study the evolution of the pandemic and the risk if travel restrictions were lifted, various vaccination strategies were implemented together, different levels of NPI containment intensities, all of which are works in progress.

\section{CONCLUSION}

To summarize, in this article we estimated a plausible lower bound on the outbreak size and sickbed demands if the pandemic response strategies in the reference countries were applied in China. The estimates revealed the real possibility of a colossal outbreak which would almost certainly induce an unaffordable burden to the medical system. Our findings have raised a clear warning that, for the time being, we are not ready to embrace "open-up" strategies resting solely on the hypothesis of herd immunity induced by vaccination advocated by certain western countries. More efficient vaccinations or more specific treatment, preferably the combination of both, are needed before entry-exit quarantine measures and other COVID-19 response strategies in China can be safely lifted.

According to the celebrated dynamic models in epidemiology and Grönwall's Inequality in math, an epidemic decays exponentially when the reproductive number $R<1$, but may also blow up in the same exponential manner once $R>1$. In the past year, many in the world have suffered by overconfidently jumping into the latter scenario. China should not, and cannot afford to, be the next.

Conflicts of interest: No conflicts of interest.

Funding: National Natural Science Foundation of China grant 8204100362 and The Bill \& Melinda Gates Foundation (INV-016826).

doi: $10.46234 / \mathrm{ccdcw} 2021.245$

\# Corresponding authors: Yuan Zhang, zhangyuan@math.pku.edu.cn; Xiaohua Zhou, azhou@math.pku.edu.cn;.

\footnotetext{
${ }^{1}$ School of Mathematical Sciences, Peking University, Beijing, China;

${ }^{2}$ Center for Statistical Sciences, Peking University, Beijing, China;

3 Beijing International Center for Mathematical Research, Peking University, Beijing, China; ${ }^{4}$ Department of Biostatistics, School of Public Health, Peking University, Beijing, China.

\& Joint first authors.
}

Submitted: October 18, 2021; Accepted: October 25, 2021

\section{REFERENCES}

1. National Bureau of Statistics of China. Communiqué of the seventh national population census (No. 3). http://www.stats.gov.cn/ english/PressRelease/202105/t20210510_1817188.html. [2021-5-11].

2. The Central People's Government of the People's Republic of China. Territory of the People's Republic of China. http://www.gov.cn/test/ 2005-06/15/content_18252.htm. [2021-8-23]. (In Chinese).

3. The Central People's Government of the People's Republic of China. Administrative divisions of the People's Republic of China. http:// www.gov.cn/test/2005-06/15/content_18253.htm. [2021-8-23]. (In Chinese)

4. The People's Government of Fujian Province. Overview of Fujian. http: //www.fujian.gov.cn/zjfj/sqgk/. [2021-8-23]. (In Chinese).

5. The Chinese Central Government's Official Website. Press Conference of the Joint Prevention and Control Mechanism of the State Council. http://www.gov.cn/xinwen/gwylflkjz164/index.htm. [2021-8-13]. (In Chinese).

6. Chinese Center for Disease Control and Prevention. Understanding of the antibody prevalence of COVID-19 in the population -- Q\&Aon the results of the nationalwide seroepidemiological survey of COVID-19. https://www.chinacdc.cn/yw_9324/202012/t20201228_223494.html. [2020-12-28]. (In Chinese).

7. The U.S. Census Bureau. Population Clock-USA and World. https:// www.worldatlas.com/geography/population-clock-usa-and-world.html. [2021-8-23].

8. Office for National Statistics, U.K. Population estimates time series dataset. https://www.ons.gov.uk/peoplepopulationandcommunity/popu lationandmigration/populationestimates/timeseries/ukpop/pop. [2021- 
8-23].

9. The Israel Central Bureau of Statistics. Monthly Bulletin of StatisticsAugust 2021. https://www.cbs.gov.il/en/publications/Pages/2021/ Monthly-Bulletin-of-Statistics-August-2021.aspx. [2021-8-23].

10. Spanish National Statistics Institute. Population figures and DemographicCensuses.https://www.ine.es/dyngs/INEbase/es/operacion. htm?c=Estadistica_C\&cid=1254736176951\&menu=ultiDatos\&idp=12 54735572981. [2021-8-23]. (In Spanish).

11. The National Institute of Statistics and Economic Studies, France. Average population of the year. https://www.insee.fr/en/statistiques/ serie/001641584. [2021-8-23].

12. United Nations. World Population Prospects 2019. https://population. un.org/wpp/Download/Standard/Population/. [2021-8-23].

13. Mathieu E, Ritchie H, Ortiz-Ospina E, Roser M, Hasell J, Appel C, et al. A global database of COVID-19 vaccinations. Nat Hum Behav 2021;5(7):947 - 53. http://dx.doi.org/10.1038/s41562-021-01122-8.

14. Dong ES, Du HR, Gardner L. An interactive web-based dashboard to track COVID-19 in real time. Lancet Inf Dis 2020;20(5):533 - 4. http: //dx.doi.org/10.1016/S1473-3099(20)30120-1.

15. Yangzhou Health Commission. The announcement on COVID-19 on
24/8/2021. http://wjw.yangzhou.gov.cn/yzwshjh/ywkd/202108/cbb092 dcf00e4e55b8827dafb451050d.shtml. [2021-8-24]. (In Chinese).

16. Yangzhou Health Commission. The announcement on COVID-19 on 16/8/2021. http://wjw.yangzhou.gov.cn/yzwshjh/ywkd/202108/2475 9ec3f09941709f39f793f17640d3.shtml. [2021-8-16]. (In Chinese).

17. National Bureau of Statistics of China. Communiqué of the seventh national population census (No. 5). http://www.stats.gov.cn/english/ PressRelease/202105/t20210510_1817190.html. [2021-5-11].

18. National Health Commission of the People's Republic of China. The Latest Situation of COVID-19 as of 24:00 on 2020-02-18. http:// www.nhc.gov.cn/xcs/yqtb/202002/8f2cfd17f4c040d89c69a4b29e 99748c.shtml. [2020-2-18]. (In Chinese).

19. De Foo C, Grépin KA, Cook AR, Hsu LY, Bartos M, Singh S, et al. Navigating from SARS-CoV-2 elimination to endemicity in Australia, Hong Kong, New Zealand, and Singapore. Lancet 2021;398 (10311):1547 - 51. http://dx.doi.org/10.1016/S0140-6736(21)021863 .

20. Kupferschmidt K. Pandemic enters transition phase-but to what? Science 2021;374(6564):135-6. http://dx.doi.org/10.1126/science. acx9290. 